\title{
Lensing function relation in Hadrons
}

\section{Simone Rodini*}

Dipartimento di Fisica, Università degli Studi di Pavia, I-27100 Pavia, Italy, Istituto Nazionale di Fisica Nucleare, Sezione di Pavia, I-27100 Pavia, Italy

E-mail: simone.rodinioleuniversitadipavia.it

\section{Barbara Pasquini}

Dipartimento di Fisica, Università degli Studi di Pavia, I-27100 Pavia, Italy, Istituto Nazionale di Fisica Nucleare, Sezione di Pavia, I-27100 Pavia, Italy E-mail: barbara.pasquini@unipv.it

\section{Alessandro Bacchetta}

Dipartimento di Fisica, Università degli Studi di Pavia, I-27100 Pavia, Italy, Istituto Nazionale di Fisica Nucleare, Sezione di Pavia, I-27100 Pavia, Italy

E-mail: alessandro.bacchetta@unipv.it

\begin{abstract}
Models provide useful insights in the partonic structure of hadrons. However, one has to use great care in the interpretation of the results when the models induce non-trivial relations between parton distributions that are independent functions in QCD. One example is the model-dependent relation between transverse distortions in the distribution of quarks in impact-parameter space and analogous distortions in transverse-momentum space due T-odd effects. We discuss the origin of such relation, pointing out the very specific conditions under which it is realised. These conditions are typically verified only in relatively simple models that describe hadrons as two-body bound systems and involve a helicity-conserving coupling between the gauge boson and the spectator system.
\end{abstract}

Light Cone 2019 - QCD on the light cone: from hadrons to heavy ions - LC2019

16-20 September 2019

Ecole Polytechnique, Palaiseau, France

\footnotetext{
${ }^{*}$ Speaker.
} 


\section{Introduction}

In QCD, we can access a three-dimensional picture of hadrons from two types of parton distributions. The first ones are impact-parameter dependent parton distributions (IPDs), which contribute to observable asymmetries in exclusive processes involving hadrons. Secondly, there are transverse-momentum dependent parton distributions (TMDs) that give rise to observable asymmetries in semi-inclusive deep inelastic (SIDIS) processes. Both distributions depend on the longitudinal momentum fraction of the partons. The additional two-dimensional information is given by the transverse parton position (or impact parameter) $\boldsymbol{b}_{\perp}$ in the case of IPDs, whereas it is given by the transverse parton momentum $\boldsymbol{k}_{\perp}$ for TMDs.

At leading twist, the IPDs quark correlator has, formally, the same structure as the quark correlator for TMDs, with $\boldsymbol{b}_{\perp}$ taking the role of $\boldsymbol{k}_{\perp}$. However, it is not possible to establish modelindependent relations between IPDs and TMDs because $\boldsymbol{b}_{\perp}$ and $\boldsymbol{k}_{\perp}$ are not conjugate variables. Non-trivial relations between the two types of distributions show up only in some model calculations $[1,2,3]$. The most prominent case is the translation of the T-even transverse-position space asymmetry, described by certain IPDs, into a T-odd transverse-momentum space asymmetry observed in SIDIS processes. This relation is established via the factorisation of the final state interactions (FSIs) in the average transverse momentum of the active quark, as described in a so-called "chromodynamics lensing function" [4, 5, 6].

In this contribution, we summarise the very stringent conditions for the validity of the lensing relation that have been discussed more extensively in Ref. [7].

\section{Lensing relation}

The quark TMD correlator is defined as

$$
\Phi^{[\Gamma]}\left(x, \boldsymbol{k}_{\perp}, S\right)=\left.\frac{1}{2} \int \frac{d z^{-} d \boldsymbol{z}_{\perp}}{(2 \pi)^{3}} e^{i k \cdot z}\left\langle p, S\left|\bar{\psi}\left(-\frac{z}{2}\right) \Gamma \mathscr{W}\left(-\frac{z}{2}, \frac{z}{2}\right) \psi\left(\frac{z}{2}\right)\right| p, S\right\rangle\right|_{z^{+}=0},
$$

where $p=\left(p^{+}, p^{-}, \boldsymbol{p}_{\perp}=\mathbf{0}_{\perp}\right)$ and $S$ are, respectively, the hadron-target momentum and spin, $\psi$ is the quark field operator, $\Gamma$ is a generic matrix in the Dirac space and $x=\frac{k^{+}}{p^{+}}$is the light-cone momentum fraction of the quark. The TMD correlator depends on $x$ and on the quark transverse momentum $\boldsymbol{k}_{\perp}$. The correlator from which the IPDs are defined reads:

$$
\mathscr{F}^{[\Gamma]}\left(x, \boldsymbol{b}_{\perp}, S\right)=\frac{1}{2} \int \frac{d z^{-}}{2 \pi} e^{i x p^{+} z^{-}}\left\langle p^{+}, \boldsymbol{R}_{\perp}=\mathbf{0}_{\perp}, S\left|\bar{\psi}\left(z_{1}\right) \Gamma \mathscr{W}\left(z_{1}, z_{2}\right) \psi\left(z_{2}\right)\right| p^{+}, \boldsymbol{R}_{\perp}=\mathbf{0}_{\perp}, S\right\rangle,
$$

where the quark fields are evaluated at $z_{1,2}=\left(0^{+}, \mp \frac{z^{-}}{2}, \boldsymbol{b}_{\perp}\right)$ and the hadron is in a state with longitudinal momentum $p^{+}$and transverse center of momentum (the infinite-momentum frame equivalent of the center of mass position) $\boldsymbol{R}_{\perp}=\mathbf{0}_{\perp}$. The operator $\mathscr{W}$, known as Wilson line or gauge link, ensures color gauge invariance of the matrix element and is defined as follows:

$$
\mathscr{W}(a, b)=\mathscr{P} \exp \left\{-i g_{s} \int_{\gamma} d \zeta \cdot A(\zeta)\right\}
$$


where $g_{s}=\sqrt{4 \pi \alpha_{s}}$ and $\gamma$ is a path from $a$ to $b$. The explicit form of the path is determined by the physical process under consideration. In the following, we will consider the gauge-link for a SIDIS process. Once the integration over $\boldsymbol{z}_{\perp}$ in Eq. (1.1) is performed, the analogy between the TMD correlator and the IPD correlator (under the exchange $\boldsymbol{k}_{\perp} \leftrightarrow \boldsymbol{b}_{\perp}$ ) becomes evident. This suggests a relation between T-odd TMDs and T-even IPDs for unpolarized (transversely) polarized quarks in a transversely polarized (unpolarized) target.

We consider the following average quark transverse momenta

$$
\left\langle k_{\perp}^{i}(x)\right\rangle_{U T}=\int d^{2} \boldsymbol{k}_{\perp} k_{\perp}^{i} \Phi^{\left[\gamma^{+}\right]}\left(x, \boldsymbol{k}_{\perp}, \boldsymbol{S}_{\perp}\right), \quad\left\langle k_{\perp}^{i}(x)\right\rangle_{T U}^{j}=\int d \boldsymbol{k}_{\perp} k_{\perp}^{i} \Phi^{\left[i \sigma^{j+} \gamma_{5}\right]}\left(x, \boldsymbol{k}_{\perp}\right),
$$

where the first and second subscripts indicate the quark and hadron polarisation, respectively. We can rewrite the $U T$ average transverse momentum as (analogous results hold for $\left\langle k_{\perp}^{i}(x)\right\rangle_{T U}^{j}$ )

$$
\begin{aligned}
& \left\langle k_{\perp}^{i}(x)\right\rangle_{U T}=\left.\frac{1}{2} \int \frac{d z^{-}}{2 \pi} e^{i x p^{+} z^{-}}\left\langle p, \boldsymbol{S}_{\perp}\left|\bar{\psi}\left(-\frac{z}{2}\right) \mathscr{W}\left(-\frac{z}{2}, \frac{z}{2}\right) \mathscr{I}^{i}\left(\frac{z}{2}\right) \gamma^{+} \psi\left(\frac{z}{2}\right)\right| p, \boldsymbol{S}_{\perp}\right\rangle\right|_{z^{+}=z_{\perp}=0} \\
& =\frac{1}{2} \int d^{2} \boldsymbol{b}_{\perp} \int \frac{d z^{-}}{2 \pi} e^{i x p^{+} z^{-}}\left\langle p^{+}, \boldsymbol{R}_{\perp}=\mathbf{0}_{\perp}, \boldsymbol{S}_{\perp}\left|\bar{\psi}\left(z_{1}\right) \mathscr{W}\left(z_{1} ; z_{2}\right) \mathscr{I}^{i}\left(z_{2}\right) \gamma^{+} \psi\left(z_{2}\right)\right| p^{+}, \boldsymbol{R}_{\perp}=\mathbf{0}_{\perp}, \boldsymbol{S}_{\perp}\right\rangle .
\end{aligned}
$$

The FSIs are encoded into the operator $\mathscr{I}^{i}(z)$ :

$$
\mathscr{I}^{i}(z)=\frac{g_{s}}{2} \int d y^{-} \mathscr{W}\left(\left(z^{-}, z^{+}, z_{\perp}\right),\left(y^{-}, z^{+}, z_{\perp}\right)\right) G^{+i}\left(y^{-}, z^{+}, z_{\perp}\right) \mathscr{W}\left(\left(y^{-}, z^{+}, z_{\perp}\right),\left(z^{-}, z^{+}, z_{\perp}\right)\right)
$$

with $G^{+i}$ being the gluon-field strength tensor. In the light-cone gauge $A^{+}=0$ with advanced boundary condition $\boldsymbol{A}_{\perp}\left(-\infty^{-}\right)=0$, one has $\mathscr{I}^{i}(z)=\frac{g_{s}}{2} A_{\perp}^{i}\left(\infty^{-}, z^{+}, \boldsymbol{z}_{\perp}\right)$ and $\mathscr{W}\left(z_{1} ; z_{2}\right)=1$. Therefore the effect of FSIs in Eq. (1.2) becomes trivial and Eq. (1.4) simplifies as

$$
\left\langle k_{\perp}^{i}(x)\right\rangle_{U T}=\left.\frac{g_{s}}{2} \int \frac{d z^{-}}{2 \pi} e^{i x p^{+} z^{-}}\left\langle p, \boldsymbol{S}_{\perp}\left|\bar{\psi}\left(-\frac{z}{2}\right) A_{\perp}^{i}\left(\infty^{-}\right) \gamma^{+} \psi\left(\frac{z}{2}\right)\right| p, \boldsymbol{S}_{\perp}\right\rangle\right|_{z^{+}=z_{\perp}=0} .
$$

One notices from Eq. (1.6) that the FSIs for the average transverse momentum in the light-cone gauge $A^{+}=0$ reduce to the exchange of a single transverse gluon at light-cone infinity between the active quark and the spectator system (the same result holds regardless of the boundary conditions for $A$ ).

The first line of Eq. (1.4) can be rewritten via the insertion of two completeness relation:

$$
\begin{aligned}
& \left\langle k_{\perp}^{i}(x)\right\rangle_{U T}=\frac{1}{2} \int\left\{d k_{1}\right\}\left\{d k_{2}\right\}\{d l\} \int \frac{d z^{-}}{2 \pi} e^{i x p^{+} z^{-}} e^{-i \frac{z^{-}}{2}\left(k_{1}^{+}+k_{2}^{+}+l^{+}\right)} \sum_{n, m} \sum_{\beta \beta^{\prime}} \int \prod_{i=1}^{n} \frac{d q_{i}^{+} d \boldsymbol{q}_{\perp, i}}{(2 \pi)^{3} 2 q_{i}^{+}} \\
& \times \prod_{j=1}^{m} \frac{d w_{j}^{+} d \boldsymbol{w}_{\perp, j}}{(2 \pi)^{3} 2 w_{i}^{+}}\left\langle p^{+}, \boldsymbol{p}_{\perp}=\mathbf{0}_{\perp}, \boldsymbol{S}_{\perp}\left|\phi\left(k_{1}\right) \gamma^{+}\right|\left\{q_{i}^{+}, \boldsymbol{q}_{\perp, i}\right\}_{n}, \beta^{\prime}\right\rangle\left\langle\left\{q_{i}^{+}, \boldsymbol{q}_{\perp, i}\right\}_{n}, \beta^{\prime}\left|I^{i}(l)\right|\left\{w_{i}^{+}, \boldsymbol{w}_{\perp, i}\right\}_{m}, \boldsymbol{\beta}\right\rangle \\
& \times\left\langle\left\{w_{i}^{+}, \boldsymbol{w}_{\perp, i}\right\}_{m}, \beta\left|\psi\left(k_{2}\right)\right| p^{+}, \boldsymbol{p}_{\perp}=\mathbf{0}_{\perp}, \boldsymbol{S}_{\perp}\right\rangle,
\end{aligned}
$$

where $\{\ldots\}$ indicates the Lorentz invariant integration measure and $\phi\left(\frac{z}{2}\right)=\bar{\psi}\left(-\frac{z}{2}\right) \mathscr{W}\left(-\frac{z}{2} ; \frac{z}{2}\right)$. In Eq. (1.7), the indices $\beta$ and $\beta^{\prime}$ label the parton, color and the helicity content of the intermediate 
states. The matrix elements of the lensing operator $I^{i}(l)$ in Eq. (1.8) represent the interaction between the active parton and the spectator system mediated by the Wilson gluons and correspond to the FSIs. To recover the IPD definition in Eq. (1.7) and, therefore, to have the factorisation of the FSIs, the operator $I^{i}(l)$ has to satisfy the following relation ${ }^{1}$ :

$$
\begin{aligned}
& \left\langle\left\{q_{i}^{+}, \boldsymbol{q}_{\perp, i}\right\}_{n}, \beta^{\prime}\left|I^{i}(l)\right|\left\{w_{i}^{+}, \boldsymbol{w}_{\perp, i}\right\}_{m}, \boldsymbol{\beta}\right\rangle \\
& =2 \pi L^{i}\left(\frac{\boldsymbol{l}_{\perp}}{1-x}\right) \delta_{n, m} \boldsymbol{\delta}_{\beta \beta^{\prime}} \boldsymbol{\delta}\left(l^{+}\right) \prod_{i=1}^{n}(2 \pi)^{3} 2 q_{i}^{+} \boldsymbol{\delta}\left(q_{i}^{+}-w_{i}^{+}\right) \boldsymbol{\delta}\left(\boldsymbol{q}_{\perp, i}-\boldsymbol{w}_{\perp, i}-x_{i} \frac{\boldsymbol{l}_{\perp}}{1-x}\right) .
\end{aligned}
$$

Eq. (1.8) holds under the following conditions for the FSIs:

1. the FSIs should connect Fock states with the same number of constituents and the same parton, helicity and color content;

2. the FSIs should transfer the total transverse momentum $l_{\perp} /(1-x)$ to the whole spectator system;

3. the FSIs can not transfer momentum in the light-cone direction to the spectator system;

4. the FSIs should transfer a fraction $x_{i}=w_{i}^{+} / p^{+}$of the total transverse momentum to each constituent of the spectator system.

The last condition is the most stringent. It is crucial to obtain the correct transverse light-front boost that gives the non-diagonal matrix element in momentum space and then the transverse distortion in impact parameter space described by the IPDs. In the light-cone gauge with advanced boundary conditions, one can easily deduce that the condition (4) can be realised via a single particle coupling (e.g. perturbative coupling) between the gauge boson and the spectator system only if the latter is composed by a single constituent, i.e. the hadron target is a two-body bound system. In this case, the light-cone momentum fraction of the spectator is equal to $(1-x)$ and the constraint on the transverse momentum transferred by the Wilson gluon to the spectator system follows trivially from the conservation of the total momentum of the hadron target. Otherwise, the condition (4) imposes to share the transverse momentum carried by the Wilson gluon with each spectator parton in a proportion equal to the longitudinal momentum fraction $x_{i}$. This cannot be realised in systems composed by more than two constituents by assuming an interaction vertex between the gauge boson and a single parton in the remnant.

We conclude that if, and only if, the above conditions are fulfilled we can write

$$
\begin{aligned}
\left\langle k_{\perp}^{i}(x)\right\rangle_{U T} & =\int d b_{\perp} \mathscr{L}^{i}\left(\frac{b_{\perp}}{1-x}\right) \mathscr{F}^{\left[\gamma^{+}\right]}\left(x, b_{\perp}, S_{\perp}\right), \\
\left\langle k_{\perp}^{i}(x)\right\rangle_{T U}^{j} & =\int d b_{\perp} \mathscr{L}^{i}\left(\frac{b_{\perp}}{1-x}\right) \mathscr{F}^{\left[i \sigma^{j+} \gamma_{5}\right]}\left(x, b_{\perp}, S_{\perp}\right) .
\end{aligned}
$$

One can check that the conditions ((1))-((4)) are verified in models that describe hadrons as a two-body system in which the remnant is either massive with spin $\leq 1 / 2$ or massless with arbitrary

\footnotetext{
${ }^{1} x_{i}$ is the light-cone momentum fraction of each constituent w.r.t. the hadron target light-cone momentum, i.e. $x_{i}=w_{i}^{+} / p^{+}$, and should satisfy the relation $\sum_{i} x_{i}=1-x$.
} 
spin. Examples of models with these features are: the quark target model [1]; for a proton target, the scalar-diquark spectator model $[1,6]$ and the axial-diquark models that admit only transverse polarization for the diquark [8]; for a pion target, relativistic models at the lowest order in the Fock-space expansion $[9,10]$. Viceversa, the conditions are not fulfilled in many-body models for hadrons, such as the three-quark model for the nucleon [11, 12, 13, 14, 15], or in two-body models in which the coupling with the Wilson gluon allows a helicity transition for the remnant, as, for example, the axial-vector diquark model with longitudinally polarised diquark.

To summarise, model calculations of TMDs play a crucial role for building educated Ansätze for fits of the TMDs and GPDs, and are essential towards an understanding of the non-perturbative aspects of TMDs. However, it should be avoided to use model-induced relations as constraint to extract information on these distributions from data. Instead, one should use the results of the extractions to understand how good the model-induced relations are, because this will eventually shed light on some of the non perturbative aspects of QCD.

\section{Acknowledgments}

This work is supported by the European Research Council (ERC) under the European Union's Horizon 2020 research and innovation programme (grant agreement No. 647981, 3DSPIN).

\section{References}

[1] S. Meissner, A. Metz and K. Goeke, Relations between generalized and transverse momentum dependent parton distributions, Phys. Rev. D76 (2007) 034002 [hep-ph / 0703176$].$

[2] H. Avakian, A. V. Efremov, P. Schweitzer and F. Yuan, The transverse momentum dependent distribution functions in the bag model, Phys. Rev. D81 (2010) 074035 [1001.5467].

[3] C. Lorcé and B. Pasquini, On the Origin of Model Relations among Transverse-Momentum Dependent Parton Distributions, Phys. Rev. D84 (2011) 034039 [1104 . 5651].

[4] M. Burkardt, Quark correlations and single spin asymmetries, Phys. Rev. D69 (2004) 057501 [hep-ph/0311013].

[5] M. Burkardt, Chromodynamic lensing and transverse single spin asymmetries, Nucl. Phys. A735 (2004) 185 [hep-ph/ 0302144$].$

[6] M. Burkardt and D. S. Hwang, Sivers asymmetry and generalized parton distributions in impact parameter space, Phys. Rev. D69 (2004) 074032 [hep-ph/ 0309072 ].

[7] B. Pasquini, S. Rodini and A. Bacchetta, Revisiting model relations between T-odd transverse-momentum dependent parton distributions and generalized parton distributions, Phys. Rev. D100 (2019) 054039 [1907.06960].

[8] A. Bacchetta, F. Conti and M. Radici, Transverse-momentum distributions in a diquark spectator model, Phys. Rev. D78 (2008) 074010 [0 807.0323 ].

[9] L. Gamberg and M. Schlegel, Final state interactions and the transverse structure of the pion using non-perturbative eikonal methods, Phys. Lett. B685 (2010) 95 [0911.1964].

[10] B. Pasquini and P. Schweitzer, Pion transverse momentum dependent parton distributions in a light-front constituent approach, and the Boer-Mulders effect in the pion-induced Drell-Yan process, Phys. Rev. D90 (2014) 014050 [1406.2056]. 
[11] B. Pasquini and F. Yuan, Sivers and Boer-Mulders functions in Light-Cone Quark Models, Phys. Rev. D81 (2010) 114013 [1001.5398].

[12] B. Pasquini, S. Cazzaniga and S. Boffi, Transverse momentum dependent parton distributions in a light-cone quark model, Phys. Rev. D78 (2008) 034025 [0806.22 98].

[13] S. Boffi, B. Pasquini and M. Traini, Linking generalized parton distributions to constituent quark models, Nucl. Phys. B649 (2003) 243 [hep-ph/0207340].

[14] B. Pasquini, M. Pincetti and S. Boffi, Chiral-odd generalized parton distributions in constituent quark models, Phys. Rev. D72 (2005) 094029 [hep-ph/ 0510376 ].

[15] B. Pasquini and S. Boffi, Nucleon spin densities in a light-front constituent quark model, Phys. Lett. B653 (2007) 23 [0 705.4345$].$ 